\title{
Cigarette and IL-17A synergistically induce bronchial epithelial-mesenchymal transition via activating IL-17R/NF-KB signaling
}

\author{
Libing Ma', Ming Jiang ${ }^{1}$, Xiaoli Zhao², Jingyi Sun ${ }^{1}$, Qilu Pan ${ }^{1}$ and Shuyuan $\mathrm{Chu}^{2^{*}}$ (1)
}

\begin{abstract}
Background: IL-17A directly induces epithelial-mesenchymal transition (EMT) in alveolar epithelial cells. It could coordinate with cigarette smoke extract (CSE) to promote proliferation of bronchial epithelial cells. In this study, we aim to explore the direct effect of IL-17A and CSE on EMT in bronchial epithelial cells.

Methods: Bronchial epithelial cells were isolated from C57BL/6 mice, and cocultured with CSE or/and IL-17A. Ecadherin and Vimentin expressions in cells were detected using immunofluorescence staining. IL-17R expression was detected using immunohistochemistry staining. NF-KB expression was assessed using western blotting. When NF-KB was inhibited by BAY 11-7821, expressions of NF-KB, E-cadherin and Vimentin were measured.

Results: The protein expression of E-cadherin in bronchial epithelial cells was lowest in CSE + IL-17A group, followed by CSE group. In contrast, the protein expression of Vimentin was highest in CSE + IL-17A group, followed by CSE group. Similarly, IL-17R and NF-KB expressions were highest in CSE + IL-17A group, followed by CSE group and IL-17A group. NF-kB inhibitor could inhibit the expressions of E-cadherin and Vimentin.

Conclusions: Cigarette and IL-17A could synergistically induce EMT in bronchial epithelial cells through activating IL17R/NF-KB signaling. Our findings contribute to a better understanding in airway EMT and pathogenesis of respiratory diseases, which are involved IL-17A and cigarette smoking. Those will provide novel avenues in the immunotherapy of lung diseases.
\end{abstract}

Keywords: Epithelial-mesenchymal transition, Bronchial epithelial cell, IL-17, NF-kB, Cigarette smoke extract

\section{Background}

Epithelial-mesenchymal transition (EMT) in bronchial epithelial cells is involved in pathogenesis of lung cancer, chronic obstructive pulmonary disease (COPD), asthma and pulmonary fibrosis, which all have a high prevalence [1-4]. IL-17A is involved in these lung diseases. This interleukin promotes chronic immune inflammation, contributing to occurrence and development of those lung diseases [5-7]. In airway, IL-17A could induce production of chemokine and cytokines from bronchial epithelial cells $[8,9]$. Interestingly, IL-17A not only promotes airway remodeling [10], but also directly induces EMT in alveolar epithelial cells [11]. That indicates a direct effect of IL-17A on

\footnotetext{
* Correspondence: emilyyuanchu@163.com

${ }^{2}$ Laboratory of Respiratory Disease, Affiliated Hospital of Guilin Medical

University, Guilin 541001, Guangxi, China

Full list of author information is available at the end of the article
}

structure cells in airway. However, there is rare report about the direct effect of IL-17A on EMT in bronchial epithelial cells. Thus, we investigated the direct effect of IL17A on EMT in murine bronchial epithelial cells.

As widely accepted, cigarette smoking is a risk factor for lung diseases, particularly lung cancer and COPD. Cigarette smoke extract (CSE) could coordinate with IL$17 \mathrm{~A}$ to induce proliferation of human bronchial epithelial cell line 16HBE [12]. CSE could significantly enhance IL17R expression in 16HBE as well [12]. Those findings suggest the synergistical effect of CSE and IL-17 on bronchial epithelial cells. Since CSE could induce EMT of bronchial epithelial cells in vitro and in vivo [13], we explored the synergistical effect of CSE and IL-17 on EMT in murine bronchial epithelial cells. Furthermore, previous study found that NF-kB activation could promote EMT in human bronchial epithelial cells [14]. 
Thus, we investigated the role of NF- $\mathrm{KB}$ signaling in the effect of CSE and IL-17 on bronchial EMT as potential mechanism in this study.

\section{Methods}

\section{Mouse cell preparation}

Female C57BL/6 mice (7-8 weeks old) were purchased from Hunan SJA Laboratory Animal Co. Ltd. (Hunan, China). They were housed in cages with free access to water and standard mouse chow. Animal protocols were approved by the Institutional Animal Care and Use Committee of Guilin Medical University and conformed to National Institutes of Health guidelines for the use of rodents. All experiments were repeated three times.

Mice were anaesthetized using $2 \%$ isoflurane inhalation and killed by cervical dislocation. The mouse bronchus was separated from lobes of lung, and then was minced to small pieces and digested by $0.05 \%$ pronase (Sigma, MA, USA) in DMEM/F12 media (Invitrogen, CA, USA) at $4{ }^{\circ} \mathrm{C}$ overnight. Digestion was stopped using FBS (Gibco, CA, USA). The bronchial epithelial cells were identified by CK-18 immunofluorescence staining.

Primary bronchial epithelial cells were cultured for $24 \mathrm{~h}$, and then cocultured with cigarette smoke extract (CSE) or/and IL-17A. CSE was prepared using a modification as previously reported [15]. In CSE group, bronchial epithelial cells were induced using 20\% CSE. In IL-17A group, the cells were induced using $50 \mathrm{ng} / \mathrm{ml}$ IL-17A (Biolegend, CA, USA). In CSE + IL-17A group, the cells were cocultured with $20 \%$ CSE and $50 \mathrm{ng} / \mathrm{ml}$ IL-17A (Biolegend, CA, USA). Cells in all groups were cultured at $37{ }^{\circ} \mathrm{C}$ with $5 \% \mathrm{CO} 2$ for $72 \mathrm{~h}$. The cells without administration of CSE or IL-17A were control group.

\section{NF-KB signaling inhibition}

NF- $\mathrm{B}$ B inhibitor, BAY 11-7821 (A4210, APEXBIO, Texas, USA), was used to inhibit NF- $\mathrm{kB}$ signaling. When primary bronchial epithelial cells were isolated and cultured for 24 h, they were cocultured with 10uM BAY 11-7821 overnight. Following that, the cells were prepared as described for IL-17A group, CSE group, CSE + IL-17A group and controls. The inhibition of NF-kB was identified using western blotting.

\section{Western blot analysis}

The bronchial epithelial cells were treated with $200 \mu \mathrm{l}$ RIPA for $10 \mathrm{~min}$ on ice, and then centrifuged at $12000 \times \mathrm{g}\left(4^{\circ} \mathrm{C}\right)$ for $15 \mathrm{~min}$. The loaded proteins $(150$ $170 \mu \mathrm{g}$ ) were separated on a $10 \%$ SDS-polyacrylamide gel electrophoresis, followed by transferring onto PVDF membranes. The samples were blocked with
TBS-Tween 20 containing $5 \%$ skim milk for $60 \mathrm{~min}$ at room temperature, followed by at $4{ }^{\circ} \mathrm{C}$ overnight, and finally $30 \mathrm{~min}$ at room temperature. The membranes were incubated with rabbit anti-mouse antibodies against mouse NF- $\mathrm{kB}(0.5 \mu \mathrm{g} / \mathrm{ml}$, ab16502; Abcam, Cambridge, UK), or mouse anti-mouse $\beta$-actin antibody (1:5000, 60,008-1-Ig; Proteintech, IL, USA) for $90 \mathrm{~min}$ at room temperature. When washed with phosphate buffered saline, they were incubated with horseradish peroxidase conjugated goat anti-rabbit (1:6000, SA00001-2; Proteintech, IL, USA) or anti-mouse antibodies (1:5000, SA00001-1; Proteintech, IL, USA) for $90 \mathrm{~min}$ at room temperature. At last, blots were developed with the ECL Plus reagents (Thermo pierce, IL, USA). The protein bands were analyzed using Quantity One software (Bio-Rad, CA, USA).

Immunohistochemistry and immunofluorescence staining For immunohistochemistry staining, the slides of cells were fixed by $4 \%$ paraformaldehyde for $30 \mathrm{~min}$ and incubated in $3 \% \mathrm{H} 2 \mathrm{O} 2$ for $10 \mathrm{~min}$ to quench endogenous peroxidase activity. The slides were incubated with primary rabbit anti-mouse antibody against IL-17R (1:50, ab180904; Abcam, Cambridge, UK) at $4{ }^{\circ} \mathrm{C}$ overnight, and then were incubated with horseradish peroxidase conjugated goat anti-rabbit IgG antibody (PV-9000, Zisbio, Beijing, China) at $37^{\circ} \mathrm{C}$ for $30 \mathrm{~min}$. After rinsing with PBS for three times, 3'3-diaminobenzidine-tetrahydrochloride was applied on the slides as a chromogen for $1-5 \mathrm{~min}$. Slides were counterstained in haematoxylin for 5-10 min. Micrographs were obtained using a microscope (BA210T, motic, Xiamen, China).

For immunofluorescence staining, the slides of cells were fixed by $4 \%$ paraformaldehyde for $30 \mathrm{~min}$, and incubated with $0.3 \%$ tritonX-100 at $37^{\circ} \mathrm{C}$ for $30 \mathrm{~min}$, then $5 \%$ bovine serum albumin. The slides were incubated with primary mouse anti-mouse Cytokeratin 18 (1:50, ab668; Abcam, Cambridge, UK), mouse antimouse E-cadherin (1:50, ab76055; Abcam, Cambridge, $\mathrm{UK}$ ), or rabbit anti-mouse antibody against Vimentin (1:50, ab92547; Abcam, Cambridge, UK) at $4{ }^{\circ} \mathrm{C}$ overnight, and then were incubated with Alexa Fluor 594conjugated Goat Anti-Mouse $\operatorname{IgG}(\mathrm{H}+\mathrm{L})(\mathrm{SA00006-3}$, Proteintech, IL, USA) or Alexa Fluor 594 -conjugated Goat Anti-Rabbit IgG(H $+\mathrm{L})(\mathrm{SA00006}-4$, Proteintech, IL, USA). Finally, slides were staining with DAPI (4', 6-diamidino-2-phenylindole) at $37{ }^{\circ} \mathrm{C}$ for $10 \mathrm{~min}$. Micrographs were taken from a microscope (BA210T, motic, Xiamen, China).

\section{Statistical analysis}

Statistical analyses were performed using SPSS 21.0 (IBM SPSS Inc., Chicago, IL, USA). $P$ values $<0.05$ 


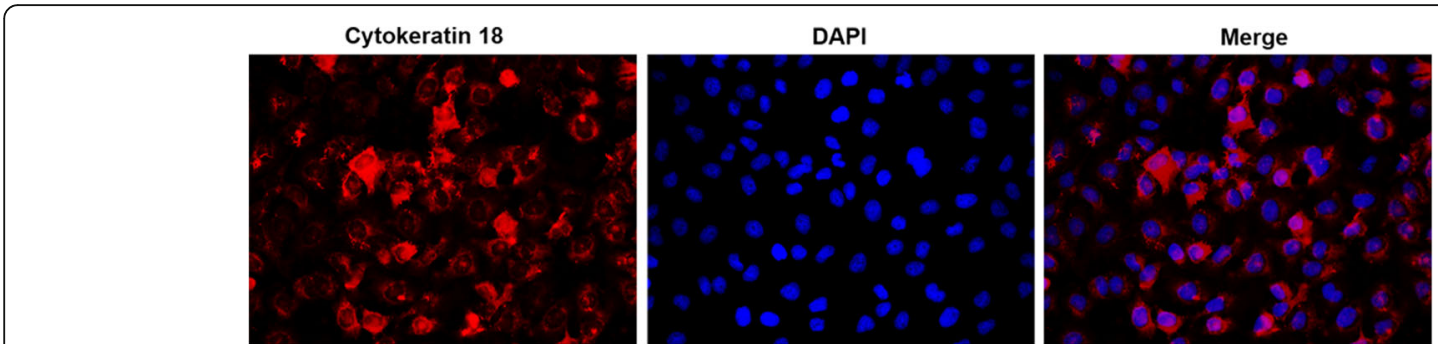

Fig. 1 Cells identification. When bronchial epithelial cells were isolated and cultured, cells were identified by immunofluorescence staining of CK-18. Cells were mainly CK-18+ staining. ( $\times 400$ magnification)

were considered to be statistically significant. Group data are expressed as the mean \pm standard deviation (SD). Significant differences were evaluated using one-way analysis of variance (ANOVA) followed by the Student-Newman-Keuls test or the GamesHowell test.

\section{Results}

Cigarette and IL-17A synergistically induce IL-17R expression in bronchial epithelial cells

Primary murine bronchial epithelial cells were identified using immunofluorescence staining of Cytokeratin18 (Fig. 1). Cytokeratin 18 is the bronchial epithelial autoantigen [16].
In murine bronchial epithelial cells, the expression of IL-17R was higher in CSE group and IL-17A group than controls. It's highest in CSE + IL-17A group (Fig. 2). These results suggest that CSE or IL-17A could induce IL-17R expression in bronchial epithelial cells. Moreover, CSE could play a synergistical role with IL-17A in inducing the IL-17R expression.

\section{Cigarette and IL-17A synergistically stimulate activation of NF-KB}

The protein expression of NF- $\mathrm{kB}$ in bronchial epithelial cells was higher in CSE group and IL-17A group than controls. It's highest in CSE + IL-17A group (Fig. 3).
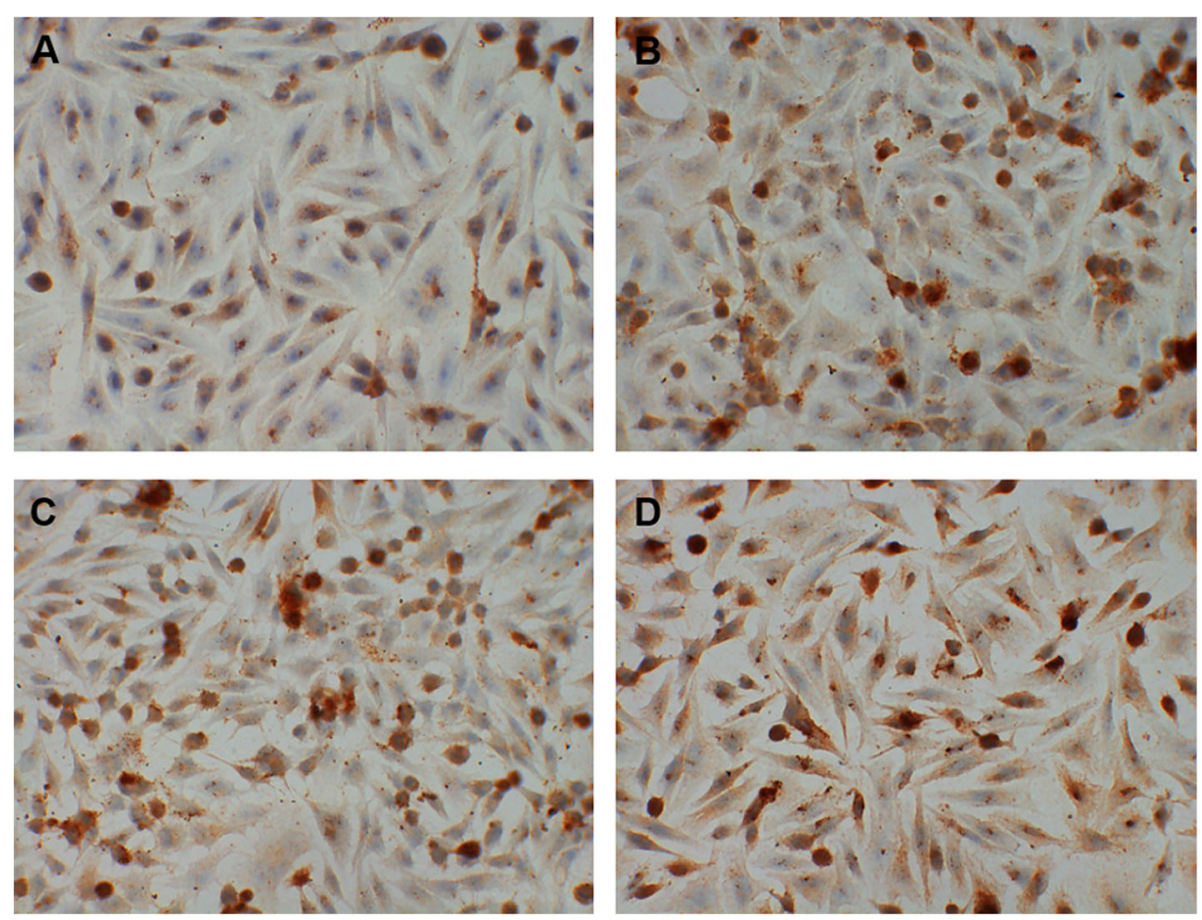

Fig. 2 IL-17R expression in bronchial epithelial cells. When bronchial epithelial cells were stimulated by cigarette smoke extract (CSE) or/and IL17A, IL-17R expression in cells were detected using immunohistochemistry staining. In CSE group and IL-17A group, IL-17R expression was increased when compared with controls. IL-17R expression was highest in CSE + IL-17A group. a control group. b CSE group. $\mathbf{c}$ IL-17A group. d CSE + IL-17A group. ( $\times 400$ magnification $)$ 


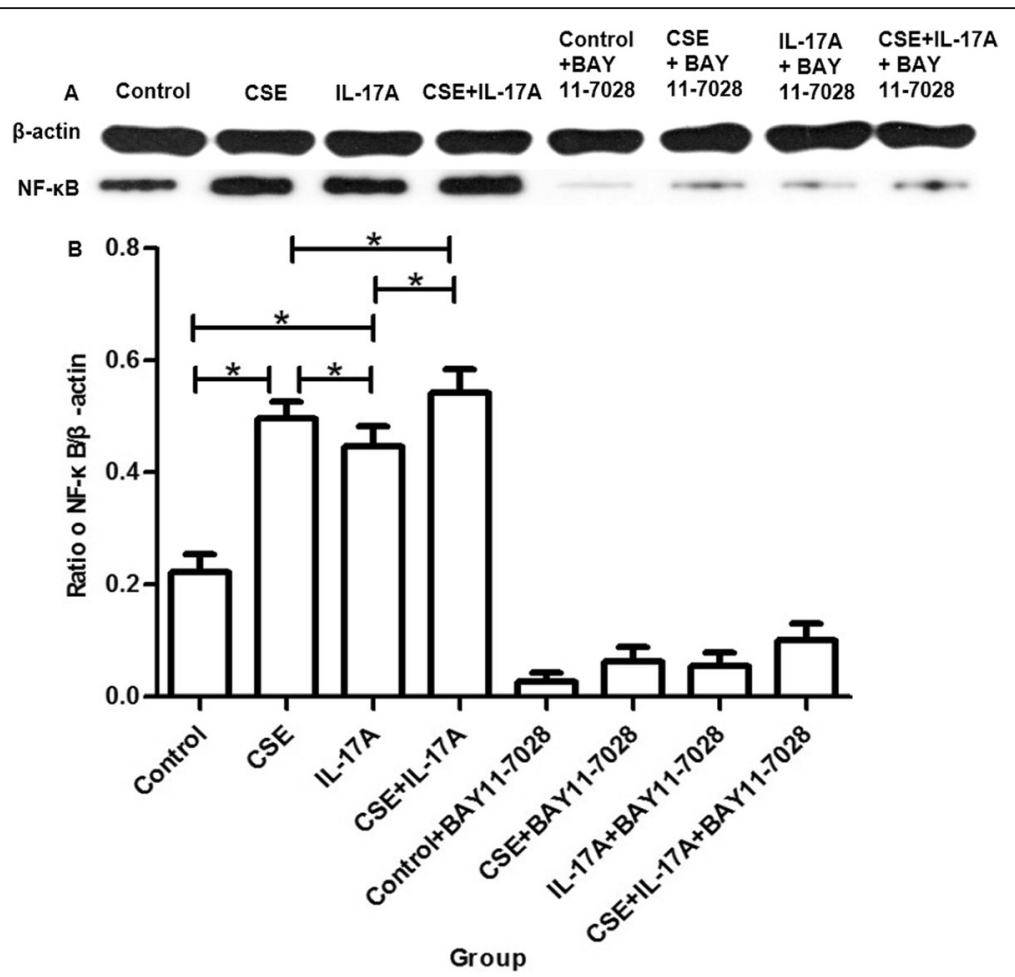

Fig. 3 The protein expression of NF-KB in bronchial epithelial cells. Bronchial epithelial cells were inhibited NF-KB, and then stimulated by cigarette smoke extract (CSE) or/and IL-17A. NF-KB expression was measured using Western blotting. In CSE group and IL-17A group, NF-KB expression was increased when compared with controls. NF-KB expression was highest in CSE + IL-17A group. When NF-KB was inhibited, NF-KB expressions in all group were significantly reduced. a Western blotting. b Quantitation of protein bands

These results suggest that NF- $\mathrm{KB}$ activation could be stimulated by CSE. And CSE could coordinate with IL-17A to stimulate NF-kB activation. When NF-kB in bronchial epithelial cells was inhibited by BAY 11-7821, NF-кB protein expression was significantly reduced (Fig. 3).

\section{Cigarette and IL-17A synergistically induce bronchial epithelial-mesenchymal transition through NF-кB signaling}

The expression of E-cadherin in bronchial epithelial cells was decreased in CSE group when compared with controls. E-cadherin expression was lowest in CSE + IL-17A group (Fig. 4a-d). In contrast, the expression of Vimentin in bronchial epithelial cells was increased in CSE group compared to controls, and was highest in CSE + IL-17A group (Fig. 5a-d). These results indicate that CSE could not only induce EMT in bronchial epithelial cells, but also act synergistically with IL-17A to promote that EMT.

When NF- $\mathrm{B}$ B was inhibited, the expressions of $\mathrm{E}-$ cadherin and Vimentin in bronchial epithelial cells were detected using immunofluorescence staining. With NF-kB inhibition, E-cadherin expression was increased in cells stimulated by CSE or CSE + IL-17A
(Fig. 4e-h) compared to those without inhibition (Fig. $4 \mathrm{a}-\mathrm{d})$. In contrast, Vimentin expression was decreased in cells stimulated by CSE or CSE + IL-17A when inhibiting NF- $\mathrm{kB}$ (Fig. 5e-h), compared to those without NF- $\mathrm{kB}$ inhibition (Fig. 5a-d). These results indicate that when NF- $\mathrm{B}$ is inhibited, the bronchial EMT is reduced. Thus, the CSE and IL-17A could synergistically induce EMT in bronchial epithelial cells through NF- $\mathrm{kB}$ signaling.

\section{Discussion}

Our study demonstrates that the protein expression of E-cadherin in bronchial epithelial cells is lowest in CSE + IL-17A group, followed by CSE group. In contrast, the protein expression of Vimentin is highest in CSE + IL17A group, followed by CSE group. Those could be inhibited by NF- $\mathrm{kB}$ inhibitor. In addition, the expressions of IL-17R and NF- $\mathrm{B}$ are similar with that of Vimentin in bronchial epithelial cells. Our results suggest that cigarette and IL-17A could synergistically induce EMT through IL17R/NF- $\mathrm{KB}$ signaling in bronchial epithelial cells.

Our results showed that in CSE group, E-cadherin expression in bronchial epithelial cells was lower than controls, whereas Vimentin expression was higher than 


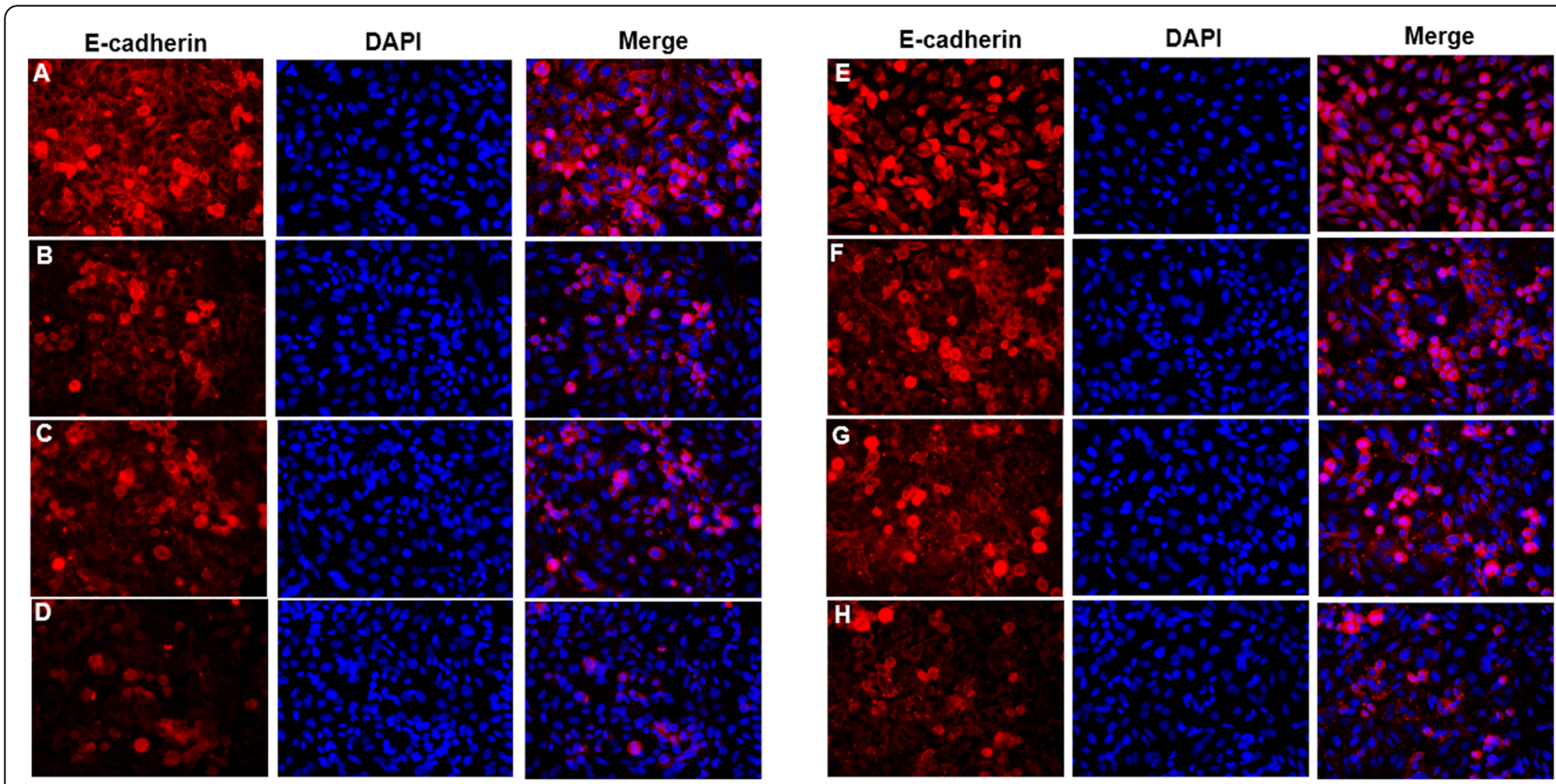

Fig. 4 E-cadherin expression in bronchial epithelial cells. When bronchial epithelial cells were stimulated with cigarette smoke extract (CSE) or/ and IL-17A, E-cadherin expression in cells was detected using immunofluorescence staining. E-cadherin expression in CSE group was lower than that in controls, and was lowest in CSE + IL-17A group. When NF-KB was inhibited, E-cadherin expression was increased in cells stimulated with CSE and CSE + IL-17A compared to those without inhibition. $\mathbf{a}$ E-cadherin expression in control group without NF-KB inhibition. $\mathbf{b}$ E-cadherin expression in CSE group without NF-KB inhibition. $\mathbf{c}$ E-cadherin expression in IL-17A group without NF-KB inhibition. $\mathbf{d}$ E-cadherin expression in CSE + IL-17A group without NF-KB inhibition. e E-cadherin expression in control group with NF-KB inhibition. $\mathbf{f}$ E-cadherin expression in CSE group with NF-KB inhibition. $\mathbf{g}$ E-cadherin expression in IL-17A group with NF-KB inhibition. $\mathbf{h}$ E-cadherin expression in CSE + IL-17A group with NF-KB inhibition. (× 400 magnification)

controls. Decreased E-cadherin expression and increased Vimentin expression could indicate EMT in bronchial epithelial cells [17]. Thus, our findings suggest that CSE could induce EMT in bronchial epithelial cells. Previous study reported that CSE could induce EMT of human bronchial epithelial cells and airway remodeling in animal model of COPD [13]. The results from our study are consistent with previous findings, and further confirm the effect of CSE on EMT in murine bronchial epithelial cells. Moreover, we found that in CSE + IL-17A group, E-cadherin expression was lower and Vimentin expression was higher than those in CSE group. Those findings indicate that cigarette and IL-17A could synergistically induce EMT in bronchial epithelial cells.

Furthermore, we explored the possible mechanism on the synergistical effect of CSE and IL-17A on EMT in bronchial epithelial cells. IL-17A could bind to IL$17 \mathrm{R}$, and induce the activation of NF- $\mathrm{KB}$ and IL-17R expression in human bronchial epithelial cells, resulting in an increased production of cytokines, such as IL-19, CXCL-2, -3 and - 5 [18]. Similarly, CSE could stimulate the activation of NF- $\mathrm{KB}$ in human bronchial epithelial cells, leading to inflammation, oxidative stress and even cell malignant transformation [19, 20]. Interestingly, in alveolar epithelial cells, CSE could coordinate with IL-17A to activate NF- $k B$, leading to inflammation [21]. Thus, we explored the role of IL$17 \mathrm{R} / \mathrm{NF}-\mathrm{kB}$ signaling in the synergistical effect of CSE and IL-17A on EMT in bronchial epithelial cells. Our study shows that IL-17R and NF- $\kappa B$ expressions in CSE group or IL-17A group were increased when compared with controls. In CSE + IL-17A group, IL$17 \mathrm{R}$ and NF- $\mathrm{KB}$ expressions were highest. Those findings confirm that CSE and IL-17A could synergistically activate IL-17R/NF- $\mathrm{kB}$ signaling in bronchial epithelial cells.

Moreover, we inhibited NF- $\mathrm{kB}$ to further demonstrate the role of NF- $\kappa B$ signaling in the effect of CSE and IL-17A on EMT in bronchial epithelial cells. Our study shows that in CSE group and CSE + IL-17 group, E-cadherin expression in bronchial epithelial cells was lower than controls, whereas Vimentin expression was higher. In CSE + IL-17E group, Ecadherin expression was lowest but Vimentin expression was highest. Those results suggest that EMT should be most aggravated when stimulated by CSE and IL-17A, followed by CSE stimulation alone. 


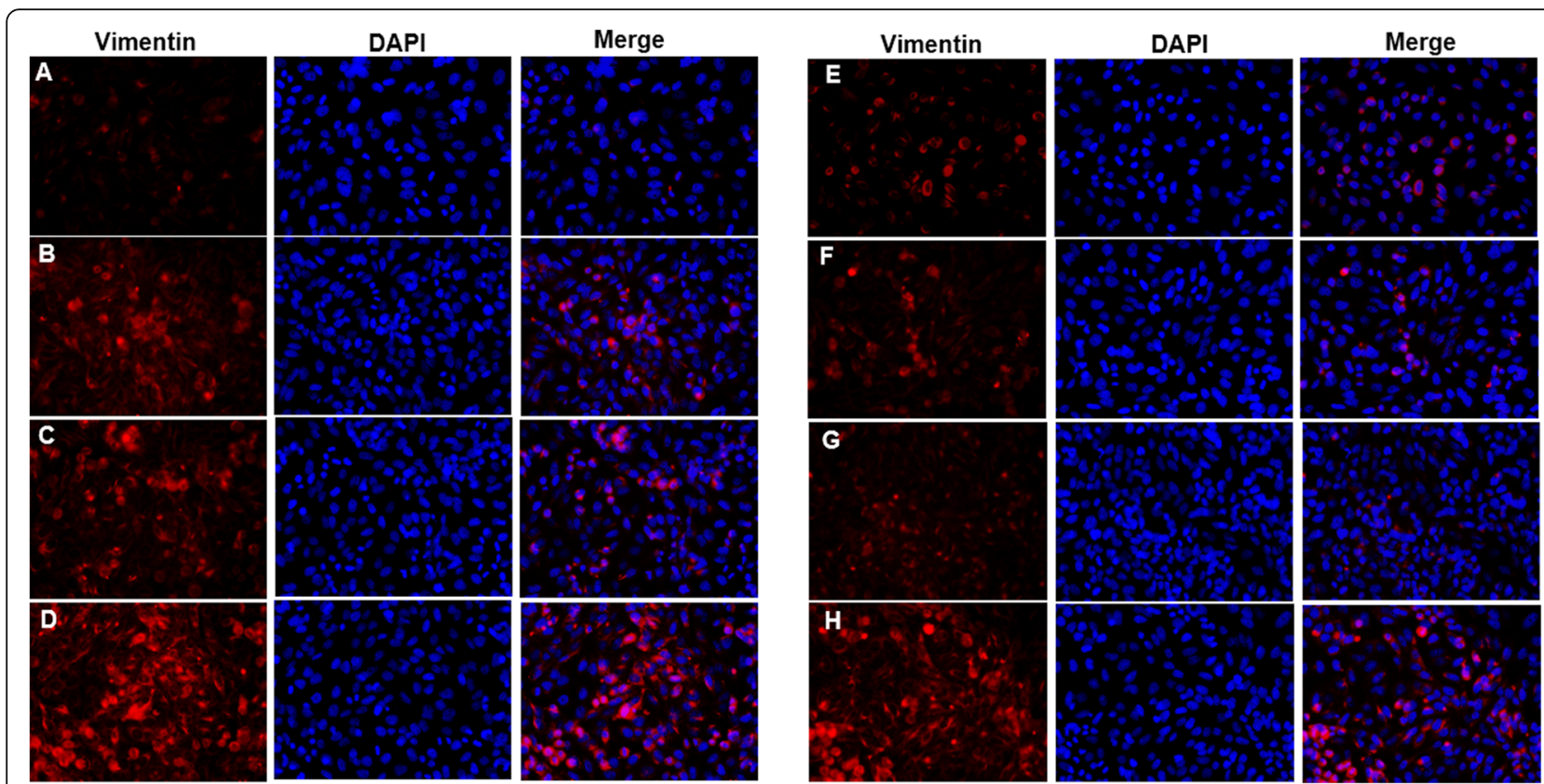

Fig. 5 Vimentin expression in bronchial epithelial cells. When bronchial epithelial cells were stimulated with cigarette smoke extract (CSE) or/ and IL-17A, Vimentin expression in cells was detected using immunofluorescence staining. Vimentin expression in CSE group was higher than that in controls, and was highest in CSE + IL-17A group. When NF-KB was inhibited, Vimentin expression was decreased in cells stimulated with CSE and CSE + IL-17A after NF-KB inhibited compared to those without inhibition. a Vimentin expression in control group without NF-KB inhibition. $\mathbf{b}$ Vimentin expression in CSE group without NF-KB inhibition. $\mathbf{c}$ Vimentin expression in IL-17A group without NF-KB inhibition. $\mathbf{d}$ Vimentin expression in CSE + IL-17A group without NF-KB inhibition. e Vimentin expression in control group with NF-kB inhibition. $\mathbf{f}$ Vimentin expression in CSE group with NF-KB inhibition. $\mathbf{g}$ Vimentin expression in IL-17A group with NF-KB inhibition. $\mathbf{h}$ Vimentin expression in CSE + IL17A group with NF-KB inhibition. ( $\times 400$ magnification)

However, the EMT is not significantly different from controls when stimulated with IL-17A alone. Thus, CSE or CSE coordinated with IL-17A may induce EMT of bronchial epithelial cells via NF- $k B$ activation. However, our study couldn't conclude that IL17A could induce EMT in murine bronchial epithelial cells via NF- $\mathrm{BB}$ activation, although IL-17A could stimulate IL-17R/NF- $\mathrm{B}$ signaling in those cells. Interestingly, an increase of IL-17A promotes COPD, asthma and lung cancer, which all have a high prrevalence [6, 7, 22]. And cigarette smoking is widely accepted as a significantly risk factor for those diseases. Thus, our study may contribute to a better understanding in the pathogenesis of those respiratory diseases, which are involved both of IL-17A and cigarette smoking.

\section{Conclusions}

Our study shows an increase of EMT in bronchial epithelial cells when stimulated with CSE, which was mostly aggravated when CSE combined with IL-17A. Similar expression pattern of IL-17R and NF- $\kappa$ B was observed. When NF- $\mathrm{BB}$ is inhibited, EMT stimulated by CSE alone or combined with IL-17A was significantly reduced. Those suggest that cigarette and IL-17A could synergistically induce EMT in bronchial epithelial cells through activating IL17R/NF- $\mathrm{B}$ signaling. Our findings contribute to a better understanding in airway EMT and the pathogenesis of respiratory diseases, which are involved with IL-17A and cigarette smoking. Those will provide novel avenues in the immunotherapy of lung diseases.

\section{Abbreviations}

ANOVA: Analysis of variance; COPD: Chronic obstructive pulmonary disease; CSE: Cigarette smoke extract; EMT: Epithelial-mesenchymal transition; SD: Standard deviation

\section{Acknowledgements}

Not applicable.

\section{Authors' contributions}

SC and LM designed the study. LM, MJ, XZ, JS and QP performed the experiment. LM and SC performed statistical analyses. LM and SC wrote the manuscript. All authors contributed to the revision and approved the final manuscript.

\section{Funding}

This work was supported by grants from the National Natural Science Foundation of China (No. 81760012), the Guangxi Natural Science

Foundation (No. 2017GXNSFBA198069). The founders do not play a role in the design of the study and collection, analysis, and interpretation of data and in writing the manuscript. 


\section{Availability of data and materials}

The datasets used and/or analyzed during the current study are available from the corresponding author on reasonable request.

\section{Ethics approval and consent to participate}

The study was approved by the Institutional Animal Care and Use Committee of Guilin Medical University. Consent to participate is not applicable.

\section{Consent for publication}

Not applicable.

\section{Competing interests}

The authors declare that they have no competing interests.

\section{Author details}

'Department of Respiratory and Critical Care Medicine, Affiliated Hospital of Guilin Medical University, Guilin 541001, Guangxi, China. ${ }^{2}$ Laboratory of Respiratory Disease, Affiliated Hospital of Guilin Medical University, Guilin 541001, Guangxi, China.

Received: 5 September 2019 Accepted: 20 January 2020

Published online: 30 January 2020

\section{References}

1. Matsubara D, Kishaba Y, Ishikawa S, Sakatani T, Oguni S, Tamura T, et al. Lung cancer with loss of BRG1/BRM, shows epithelial mesenchymal transition phenotype and distinct histologic and genetic features. Cancer Sci. 2013;104:266-73.

2. He S, Chen D, Hu M, Zhang L, Liu C, Traini D, et al. Bronchial epithelial cell extracellular vesicles ameliorate epithelial-mesenchymal transition in COPD pathogenesis by alleviating M2 macrophage polarization. Nanomedicine. 2019;18:259-71.

3. Yang ZC, Yi MJ, Ran N, Wang C, Fu P, Feng XY, et al. Transforming growth factor- $\beta 1$ induces bronchial epithelial cells to mesenchymal transition by activating the snail pathway and promotes airway remodeling in asthma. Mol Med Rep. 2013:8:1663-8.

4. Kanemaru R, Takahashi F, Kato M, Mitsuishi Y, Tajima K, Ihara H, et al. Dasatinib suppresses TGF $\beta$-mediated epithelial-Mesenchymal transition in alveolar epithelial cells and inhibits pulmonary fibrosis. Lung. 2018;196:531-41.

5. Zhang J, Chu S, Zhong X, Lao Q, He Z, Liang Y. Increased expression of CD4+IL-17+ cells in the lung tissue of patients with stable chronic obstructive pulmonary disease (COPD) and smokers. Int Immunopharmacol. 2013;15:58-66

6. Ito JT, Cervilha DAB, Lourenço JD, Gonçalves NG, Volpini RA, Caldini EG, et al. Th17/Treg imbalance in COPD progression: a temporal analysis using a CS-induced model. PLoS One. 2019;14:e0209351.

7. Camargo LDN, Righetti RF, Aristóteles LRCRB, Dos Santos TM, de Souza FCR, Fukuzaki S, et al. Effects of anti-IL-17 on inflammation, remodeling, and oxidative stress in an experimental model of asthma exacerbated by LPS. Front Immunol. 2018;8:1835.

8. Luo J, An X, Yao Y, Erb C, Ferguson A, Kolls JK, et al. Epigenetic regulation of IL-17-induced chemokines in lung epithelial cells. Mediators Inflamm. 2019; 2019:9050965.

9. Honda K, Wada H, Nakamura M, Nakamoto K, Inui T, Sada M, et al. IL-17A synergistically stimulates TNF-a-induced IL-8 production in human airway epithelial cells: a potential role in amplifying airway inflammation. Exp Lung Res. 2016;42:205-16.

10. Chakir J, Shannon J, Molet S, Fukakusa M, Elias J, Laviolette M, et al. Airway remodeling-associated mediators in moderate to severe asthma: effect of steroids on TGF-beta, IL-11, IL-17, and type I and type III collagen expression. J Allergy Clin Immunol. 2003;111:1293-8.

11. Vittal R, Fan L, Greenspan DS, Mickler EA, Gopalakrishnan B, Gu H, et al. IL-17 induces type $V$ collagen overexpression and EMT via TGF- $\beta$-dependent pathways in obliterative bronchiolitis. Am J Physiol Lung Cell Mol Physiol. 2013:304:L401-14.

12. Montalbano AM, Riccobono L, Siena L, Chiappara G, Di Sano C, Anzalone G, et al. Cigarette smoke affects IL-17A, IL-17F and IL-17 receptor expression in the lung tissue: ex vivo and in vitro studies. Cytokine. 2015;76(2):391-402.

13. Xia $H$, Xue J, Xu H, Lin M, Shi M, Sun Q, et al. Andrographolide antagonizes the cigarette smoke-induced epithelial-mesenchymal transition and pulmonary dysfunction through anti-inflammatory inhibiting HOTAIR. Toxicology. 2019;422:84-94.

14. Itoigawa Y, Harada N, Harada S, Katsura Y, Makino F, Ito J, et al. TWEAK enhances TGF- $\beta$-induced epithelial-mesenchymal transition in human bronchial epithelial cells. Respir Res. 2015;16:48.

15. Chen ZH, Kim HP, Sciurba FC, Lee SJ, Feghali-Bostwick C, Stolz DB, et al. Egr1 regulates autophagy in cigarette smoke-induced chronic obstructive pulmonary disease. PLoS One. 2008:3:e3316.

16. Nahm DH, Lee YE, Yim EJ, Park HS, Yim H, Kang Y, et al. Identification of cytokeratin 18 as a bronchial epithelial autoantigen associated with nonallergic asthma. Am J Respir Crit Care Med. 2002;165:1536-9.

17. Nishioka M, Venkatesan N, Dessalle K, Mogas A, Kyoh S, Lin TY, et al. Fibroblast-epithelial cell interactions drive epithelial-mesenchymal transition differently in cells from normal and COPD patients. Respir Res. 2015;16:72.

18. Huang $F$, Kao CY, Wachi $S$, Thai $P$, Ryu J, Wu R. Requirement for both JAKmediated PI3K signaling and ACT1/TRAF6/TAK1-dependent NF-kappaB activation by IL-17A in enhancing cytokine expression in human airway epithelial cells. J Immunol. 2007;179:6504-13.

19. Wang M, Chen X, Jin W, Xu X, Li X, Sun L. Ginsenoside Rb3 exerts protective properties against cigarette smoke extract-induced cell injury by inhibiting the p38 MAPK/NF-KB and TGF-B1NEGF pathways in fibroblasts and epithelial cells. Biomed Pharmacother. 2018;108:1751-8.

20. Lu L, Xu H, Yang P, Xue J, Chen C, Sun Q, et al. Involvement of HIF-1 aregulated miR-21, acting via the Akt/NF-kB pathway, in malignant transformation of HBE cells induced by cigarette smoke extract. Toxicol Lett. 2018:289:14-21.

21. Kaur G, Bagam P, Pinkston R, Singh DP, Batra S. Cigarette smoke-induced inflammation: NLRP10-mediated mechanisms. Toxicology. 2018;398-399:52-67.

22. Mei J, Liu L. Role of interleukin 17 in lung carcinogenesis and lung Cancer progression. Zhongguo Fei Ai Za Zhi. 2016;19:46-51.

\section{Publisher's Note}

Springer Nature remains neutral with regard to jurisdictional claims in published maps and institutional affiliations.

Ready to submit your research? Choose BMC and benefit from

- fast, convenient online submission

- thorough peer review by experienced researchers in your field

- rapid publication on acceptance

- support for research data, including large and complex data types

- gold Open Access which fosters wider collaboration and increased citations

- maximum visibility for your research: over $100 \mathrm{M}$ website views per year

At $\mathrm{BMC}$, research is always in progress.

Learn more biomedcentral.com/submissions 